\title{
Außerordentliche Tagung der Wiener ophthalmologischen
} Gesellschaft

Die Wiener ophthalmologische Gesellschaft beabsichtigt, Anfang August des Jahres 1921 eine außerordentliche Tagung zu veranstalten und lädt hiermit alle befreundeten Fachgenossen ein, daran teilzunehmen. Über die näheren Einzelheiten gibt nachstehende Mitteilung weitere Kenntnis:

Wiener ophthalmologische Gesellschaft. Außerordentliche Tagung vom 4. bis 6. August 1921.

Donnerstag, den 4. August, vormittags 9 Uhr: I. wissenschaftliche Sitzung, gewidmet der Aussprache über: Die Wirksamkeit der neueren Glaukomoperationen im Vergleiche zur klassischen Iridektomíe nach v. Graefe, Die einleitenden Vorträge haben die Herren Uhthoff über den klinischen Teil und Wessely über den theoretischen und anatomischen Teil freundlichst übernommen. Die Herren Kollegen, die sich an der Aussprache beteiligen wollen, werden gebeten, dies beim Schriftführer Doz. Dr. Lauber schriftlich anzumelden.

Donnerstag, den 4. August, nachmittags 3 Uhr: Demonstrationssitzung.

Freitag, den 5. August, vormittags 9 Uhr: II. wissenschaftliche Sitzung. Nachmittags 3 Uhr: III. wissenschaftliche Sitzung.

Samstag, den 6. August, vormittags 9 Uhr: IV. (letzte) wissenschaftliche Sitzung.

Der Ort der Sitzungen wird rechtzeitig bekannt gegeben werden. Ein Projektionsapparat und eine genügende Anzahl von Mikroskopen stehen zur Verfügung.

Vorträge und Demonstrationen sind unter genauer Angabe des Stoffes so bald als möglich, spätestens bis 15. Juni bei dem Schriftführer der außerordentlichen Tagung, Doz. Dr. E. Lauber, Wien VIII, Alserstraße 25, anzumelden. Später angemeldete Vorträge können nicht mehr auf die Tages-ordnung gesetzt werden.

Die Zeit für einen Vortrag ist auf 15 Minuten, für eine Demonstration auf 5 Minuten festgesetzt. Es gilt als selbstverstandiich, daß der Inhalt der Vorträge nicht schon früher veröffentlicht sein darf. Die Urschrift der Vorträge und der Bemerkungen bei der Aussprache, sowie Vorlagen zu ein-fachen Abbildungen sind noch vor Schluß der Tagung druckfertig an den Schriftführer abzugeben.

Der Mitgliedsbeitrag für die nichtösterreichischen Teilnehmer an der Tagung beträgt 20 Mark. Es wird ersucht, diesen Betrag an die B,heinische

56

Literatur-Verzeichuis.

Kreditbank Filiale Heidelberg nnter Konto Prof. Dr. J. Meller bis znm 15. Juni 1921

einzusenden.

Der Bericht über die Tagung wird von der Verlagsbuchhandlung den Mitgliedern nm einen um 20\% gegenüber dem Ladenpreis ermäßigten Preis znr Verfügung gestellt werden.

Dringená empfehlenswert ist die frühzeitige Anmeldnng der Teilnehmer bei dem Obmann des Wohnungsausschusses Dr. R. Kramer, Wien VIII, Kochgasse 25, da nnr durch persönliche 
Vermittlung Wohnungen zn be-scbaffen sind. Es wird ersucht, anzugeben, ob Zimmer mit einem oder zwei Betten erwünscht sind. Herr Dr. R. Kramer wird in alien die Woh-nungsfrage betreffenden Angelegenheiten gern Auskunft geben.

Wien, am 7. Jannar 1921.

I. A. der Wiener opbtbalmologischen Gresellschaft: Prof. Dr. J. Meller.

LHepatup - Verzeichnis.

Anatomie - Physiologie - Pathologic Beckers, Über Myopie bei Tieren. Berl. klin. Woch. Nr.

42. Bron, J., et Putot, B., Lesions oculaires par gaz. Arch. d'Ophthalm. 37. Bd., H. 8. Bruckner, A., und Meißner, W., Grundriß der Augenheílkunde. Leipzig,

G-. Thieme. Brunetíère, Lesions oculaires produites par les gaz asphyxiants. Arch. d'Oph-

talm. 37. Bd., 8. H. Dittmers, Fr., Über die Abhängigkeit der Unterschiedsschwelle fur

Helligkeiten

von der antagonistischen Induktion. Ztschr. f. Sinnesphysiol. 51. Bd.,

H. 3-5. v. Dungern, E., Die Schichtungstheorie des Farbensehens. Arch. f. Ophthalm.

102. Bd., 3. u. 4. H. Eppenstein, Arthur, Untersuchungen über Dehnungsfestigkeit der

elastischen

Elemente des menschlichen Augapfels. Arch. f. Ophthalm. 102. Bd.,

3. u. 4. H. Fraenkel, Eugen, Über Augenerkrankungen bei Grippe. Dt. med. Woch.

Nr. 43. Fröbes, Josef, Aus der Vorgeschichte der physiologischen Optik. Ztschr. f.

Psychol. 85. Bd., H. 1-4. Giffo, Felix, Sur un cas de migraine ophtalmoplégique. Arch.

d'Ophtalm.

37. Bd., Nr. 9. Gilbert, W., Zur Anatomie der myopischen Augenhintergrundsveränderungen.

Arch. f. Augenh. 86. Bd., 3. u. 4. H. Guillaume, A.G., Les reflexes oculocardiaques et les reflexes vago-sympathiques:

Que peut-on attendre d'eux en clinique ? Pr. méd. Nr. 59. Guttmann, Alfred, Über

Abweichungen im zeitlichen Verlauf der Nachbilder

bei verschiedenen Typen des Farbensinns. Ztschr. f. Sinnesphysiol.

51. Bd., H. 3-5. Derselbe, Die Lokalisation des Farbenkontrastes beim anomalen Trichromaten.

Ztschr.f. f. Sinnesphysiol. 51. Bd.

H. 3-5. Hedinger

E.

und Vogt

A.

Klinische und anatomische Beobachtungen über Faltung der Hornhaut

der Linsenkapsel und der Retinaoberfläche. Arch.

f. Ophthalm. 102. Bd.

3. u. 4. H. 